COMMENT. The incidence of neuromuscular weakness in critically ill children in an ICU is infrequent and develops mainly in older children, over 10 years of age, and especially in transplant recipients.

\title{
CEREBRAL PALSY
}

\section{CORD SERUM INFLAMMATORY CYTOKINES AS BIOMARKERS OF CEREBRAL PALSY}

The relationship between 78 inflammatory protein mediators in cord serum and cerebral palsy (CP) in 41 term and preterm children was determined at the University of Oulu. Finland. Infants were born in the year 1992-1993 at 4 university hospitals, and CP was diagnosed by child neurologists by age 5, according to standard criteria: a persistent abnormality of muscle tone, movement, and posture with functional impairment due to a nonprogressive lesion of the immature brain. Children with congenital cerebral malformation, prenatal viral or protozoal infection, post-neonatal brain damage, or chromosomal abnormality were excluded. Of 41 children with CP in the study group, 22 had spastic diplegia, 12 spastic hemiplegia, 3 spastic quadriplegia, and 4 dystonic quadriplegia. One paired control matched for gestational age and without $\mathrm{CP}$ was selected for each case.

In children with $\mathrm{CP}$, the levels of 8 cytokines, 1 growth factor, and 3 chemokines were higher $(\mathrm{p}<0.05)$ than in controls, findings consistent with an infection or fetal inflammatory response. Infants developing $\mathrm{CP}$ had an altered serum profile of specific protein mediators. Prematures had high IL-6 and IL-8 levels, and 9 additional cytokines showed significant changes. Several of these regulatory proteins found in newborns with $\mathrm{CP}$ are distinct from those with acute neonatal disease not resulting in $\mathrm{CP}$. Inflammatory mediators and growth factors in cord blood of CP infants are indicative of a fetal response to a pre- or perinatal infection that manifests after birth as permanent brain damage. (Kaukola T, Satyaraj E, Patel DD et al. Ann Neurol February 2004;55:186-194). (Respond: Mikko Haliman MD, PhD, Department of Pediatrics and Biocenter of Oulu, University of Oulu, PO Box 5000, FIN-90014 Oulu, Finland).

COMMENT. In references cited by the authors, chorioamnionitis is considered a risk factor for cerebral palsy (Wu YW, Colford JM. JAMA 2000;284:1417-1424); and an increase in proinflammatory cytokines in amniotic fluid or cord blood has been associated with the development of CP (Nelson KB et al. Ann Neurol 1998;44:665-675). The Finnish investigators propose that susceptible infants have an abnormal pattern of cytokines and growth factors that predispose to $\mathrm{CP}$ during perinatal infection and lead to neuronal and neuroglial brain damage. An editorial (Dammann, Leviton A. Ann Neurol Feb 2004;55:158161) cautions that the study group is small, and no appreciable differences are demonstrated between cases and controls in the distribution of the "classic" proinflammatory cytokines (eg. IL-1B, IL-6, tumor necrosis factor-a). More studies of developmental regulation of biomarkers are required to adjust for gestational age-specific changes. 
Cytokines and SIDS. Pro-inflammatory cytokines are found in the brainstem of infants dying from SIDS, and an increase in IL-6 has been demonstrated in the CSF. The limitations of these findings in the etiology of SIDS is discussed by Waters KA. (Lancet Neurology February 2004;3:81).

\section{NEONATAL CEREBRAL INFARCTION AND NEUROMOTOR DYSFUNCTION}

Twenty-two children with cerebral infarction on neonatal MRI were examined neurologically at school age in a study at Imperial College School of Medicine, Hammersmith, London, UK. Six (30\%) had hemiplegia and an additional $7(30 \%)$ had neuromotor abnormalities, including asymmetries. The remaining 9 had normal motor function. Hemiplegia occurred only in patients showing neonatal MRI evidence of hemisphere, internal capsule, and basal ganglia involvement. (Mercuri E, Barnett A, Rutherford $\mathrm{M}$ et al. Neonatal cerebral infarction and neuromotor outcome at school age. Pediatrics January 2004;113:95-100). (Respond: Eugenio Mercuri MD, Department of Paediatrics, Imperial College School of Medicine, Hammersmith, London, UK).

COMMENT. In children suffering a neonatal cerebral infarction, signs of neuromotor impairment become more obvious at school age when the neurologic examination is more structured.

Risk of recurrent stroke in children and antiphospholipid screening are reviewed from the University of California, San Francisco (Fullerton HJ, von Scheven E. Editorial. Neurology January (2 of 2);62:172-173; and Lanthier S et al. Neurology 204;62:194-200). Lanthier et al found no difference in recurrence rates between the anticardiolipin antibody (aCL)-positive and aCL-negative groups, when 185 children with a first arterial ischemic stroke or TIA were followed for a median of 3 years. However, aCL-positive children were more likely to be treated with antithrombotic agent, and treated patients were less likely to have a recurrence. In the absence of a prospective randomized trial, screening for antiphospholipid syndrome (APS) should continue as a routine component of the evaluation of arterial ischemic stroke and risk of recurrence.

Subsequent publication of the results of the antiphospholipid antibodies and stroke study (APASS), a prospective double-blind cohort study involving 1770 participants from multiple US clinical sites (JAMA Feb 4, 2004;291:576-584), concludes that the presence of $\mathrm{aCL}$ in patients with ischemic stroke does not predict an increased risk of subsequent stroke. Routine screening for aPL in patients with ischemic stroke may not be warranted. The debate continues.

\section{SEIZURE DISORDERS}

\section{EFFECTS OF AEDS ON SERUM NITRITE AND NITRATE LEVELS}

Serum nitrite and nitrate levels were determined in 34 epileptic children treated with valproic acid and 23 with carbamazepine and compared to 38 non-active epileptic children 